\title{
A translational consideration of intercellular adhesion molecule-1 biology in the perioperative setting
}

\author{
Emily Greenwald ${ }^{1}$, Koichi Yuki ${ }^{2}$ \\ ${ }^{1}$ Department of Anesthesiology, Perioperative and Pain Medicine, Boston Children's Hospital; Brandeis University \\ ${ }^{2}$ Department of Anesthesiology, Perioperative and Pain Medicine, Boston Children's Hospital
}

\begin{abstract}
Intercellular adhesion molecule-1 (ICAM-1) is a critical adhesion molecule involved in leukocyte recruitment. Since its discovery in 1986, a large number of studies have been performed to elucidate its role in vitro and in vivo. Here, we review its role in leukocyte recruitment and consider future steps to take that will enhance our understanding of ICAM-1 biology and its translational application in the perioperative setting.
\end{abstract}

Key words: Intercellular adhesion molecule, leukocyte, recruitment.

\section{Introduction}

Our body has multi-layered defense mechanisms against the outside world. Skin, for example, is the most exposed organ of the human body and acts as the first line of defense against foreign organisms present in the environment. Mucosa also acts as a strong barrier. Surgical procedures often disrupt these barriers and introduce various organisms to otherwise sterile sites. Adequate immune function to eradicate these organisms is critical for minimizing perioperative complications.

Leukocytes are major constituents in the immune system and are recruited to contaminated sites to fight against a variety of organisms that may otherwise contribute to increased morbidities and mortalities in the perioperative period. Tissue injury as a result of surgery also triggers acute inflammation to which leukocytes respond, known as 'sterile inflammation'. The mechanism by which these leukocytes migrate to the site of infection or injury is complex and involves multiple molecules. A sophisticated interaction between circulating leukocytes and the endothelium is an essential component of this response. [1] Intercellular adhesion molecule-1 (ICAM-1) is considered as one of the most critical molecules in leukocyte-endothelium interaction. Here, we review ICAM-1 biology in the context of leukocyte function and discuss its translational application in the perioperative setting.

\section{The discovery of ICAM-1 and its various forms}

ICAM-1 was discovered in 1986 as a binding partner for the adhesion molecule leukocyte function-associated antigen-1 (LFA-1). [2] ICAM-1 is encoded by seven exons; the coding region for the human ICAM1 gene spans 1599 base pairs, while the mouse gene spans 1614 base pairs. The homology between human and mouse ICAM-1 nucleotide sequences is $65 \%$ [* homology matching was performed using Genetyx-Mac software]. The amino acid sequence homology is $55 \%$, [3] suggesting a high similarity. ICAM-1 is a member of the immunoglobulin supergene family and consists of five homologous immunoglobulin-like

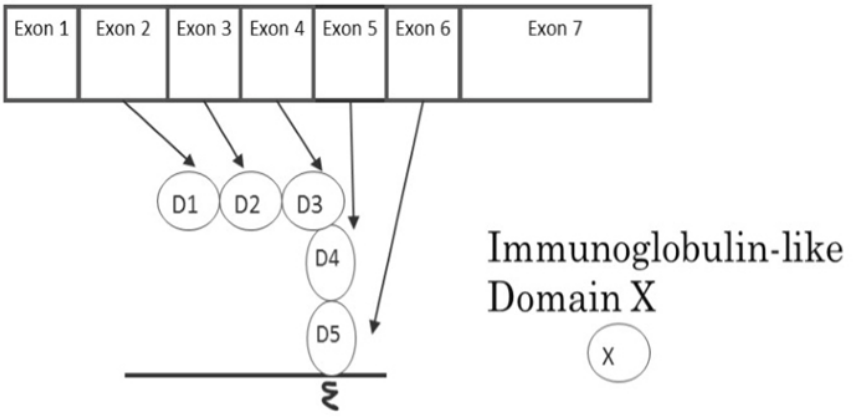

Figure 1. The relationship between ICAM-1 exons and immunoglobulin-like domains. 
domains (D1-D2-D3-D4-D5), one transmembrane domain, and a short cytoplasmic tail. Each immunoglobulin-like domain is encoded by an exon of the ICAM-1 gene, beginning with exon 2. Exon 2 encodes domain 1 (D1), and exon (X) encodes domain (X-1) until exon 6 that encodes domain 5 (D5) (Figure 1). Normally, endothelial cells, monocytes and lymphocytes have low ICAM-1 expression, but pro-inflammatory stimuli such as TNF- $\alpha$, IL-1 and IFN- $\gamma$ can upregulate its expression level. [3] Thus, ICAM-1 can be categorized as a pro-inflammatory molecule. Surgical procedures often induce pro-inflammatory responses and likely upregulate ICAM-1 expression on the endothelium.

ICAM-1 undergoes alternative splicing and post-translational modifications, and therefore exists in various forms. In mice, ICAM-1 is primarily expressed in the full-length form encoded by all of the exons and contains D1D2D3D4D5, but alternatively spliced isoforms that encode only a part of the immunoglob-
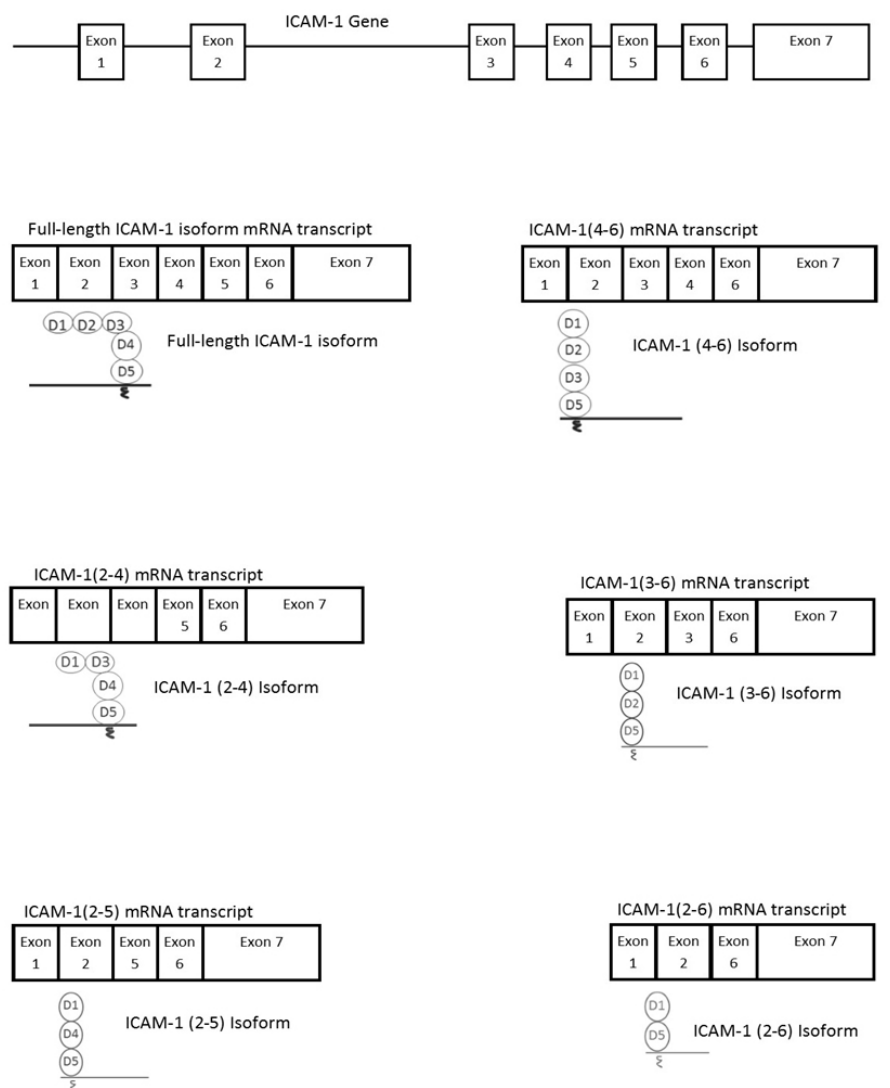

Figure 2. ICAM-1 isoforms

ulin-like domains are also produced. [4] These isoforms are named D1D2D3D5, D1D3D4D5, D1D2D5, D1D4D5 and D1D5, based on the domains that they contain (Figure 2). These forms of ICAM-1 are membrane-bound. In humans, conclusive evidence of ICAM-1 isoforms has not yet been reported. However, Dustin and collaborators reported two discrete bands specific to ICAM-1 in their study, which may suggest the presence of ICAM-1 isoforms in humans .[5] Both human and mouse ICAM-1 undergo glycosylation. It is well known that ICAM-1 is expressed on the cell surface as the membrane-bound form, and proteolytic cleavage of membrane-bound ICAM-1 (mICAM-1) produces the soluble ICAM-1 (sICAM-1). [3, 6] The function of membrane-bound and soluble ICAM-1 is described in the following section.

\section{ICAM-1 function}

\section{Membrane-bound ICAM-1 (mICAM-1)}

The function of ICAM-1 is largely studied on fulllength mICAM-1. In addition to LFA-1, mICAM-1 binds to macrophage-1 antigen (Mac-1) and aXb2 (p150,90). LFA-1, Mac-1 and aXb2 belong to the adhesion molecule family known as b2 integrins. These adhesion molecules are expressed only in leukocytes. The importance of these b2 integrins is well appreciated in a genetic disease called Leukocyte Adhesion Deficiency Type I (LAD-1); LAD-1 is caused by the loss of expression or function of b2 integrins and is characterized by impaired neutrophil recruitment and recurrent soft tissue infections, leading into death sometimes. [7, 8] As seen in a sepsis model using full-length mICAM-1 deficient mice, the lack of mICAM-1 expression impaired neutrophil recruitment to the liver, suggesting that mICAM-1 also plays a critical role in neutrophil recruitment. [9] The interaction of mICAM-1 with these molecules clearly affects the function of leukocytes.

The binding of $b 2$ integrins to mICAM-1 depends on dynamic, structural changes of the integrins from the resting conformation to the active conformation in response to activation signals. The $b 2$ integrins bind to mICAM-1 only in their active conformation. $[10,11]$ Pro-inflammatory cytokines and chemoattractants trigger intracellular signaling by interacting with leukocytes, and activate b2 integrins (inside-out signaling). In the early perioperative period, strong pro-inflammatory signals allow for appropriate activation of $\beta 2$ integrins, which is necessary for an adequate immune 
response.

LFA-1 and Mac-1 are studied most among b2 integrins. LFA-1 is expressed on all leukocytes, while Mac-1 is predominantly expressed on neutrophils, monocytes, and macrophages. The expression level of aXb2 in neutrophils is low, but monocytes and macrophages highly express aXb2. [12] Because neutrophils are the first-line defense cells in innate immunity and most relevant to perioperative immunity, we will focus on LFA-1 and Mac-1, the major b2 integrins that are expressed on neutrophils. LFA-1 and Mac-1 bind to mICAM-1 at different domains: LFA-1 binds to D1 of mICAM-1, whereas Mac-1 binds to D3 (Figure 2). Theoretically, it is possible for LFA-1 and Mac-1 to bind to mICAM-1 simultaneously because the ICAM1 domains to which each adhesion molecule binds are sufficiently far apart. However, this has not been investigated in an experimental setting. In addition, there is a difference in binding affinity of these two integrins to mICAM-1. The binding affinity between mICAM-1 and Mac-1 is weaker than that between mICAM-1 and LFA-1.[13] Full-length mICAM-1 exists primarily as a dimer on the cell surface. The binding of LFA-1 to mICAM-1 becomes two-fold stronger when mICAM-1 is present as a dimer, presumably because dimerization allows mICAM-1 to properly orient on the cell surface.[14] However, the comparison between the binding of Mac-1 to the ICAM-1 dimer and that of the monomer has not yet been reported. It is unclear if dimerization is responsible for the difference in binding affinity between LFA-1 and Mac-1. Glycosylation also plays a role in function by affecting the binding ability of Mac-1 to mICAM-1. [15] mICAM-1 likely undergoes different types of glycosylation in various tissues. It is likely that Mac-1 binds to mICAM-1 with different affinities in different tissues.

Although both LFA-1 and Mac-1 bind to mICAM-1, they may have different functional roles in leukocyte recruitment. Some leukocytes, such as the majority of T cells and B cells, express only LFA-1, while neutrophils, for example, express both. This difference in tissue distribution of b2 integrin expression accounts for the functional difference of these integrins. Both LFA-1 and Mac-1 are highly expressed in neutrophils. During recruitment, neutrophils undergo various steps: capture, rolling, slow rolling, arrest (adhesion) and transmigration. On endothelium in which inflammation has been stimulated by TNF- $\alpha$, the slow rolling of neutrophils was predominantly dependent on Mac-1: mICAM-1 interaction. [16] LFA-1 plays a significant role in neutrophil adhesion , $[17,18]$ but Dunne et al. suggested that ICAM-1 is not a ligand for LFA-1 in neutrophil adhesion to TNF- $\alpha$-stimulated endothelium. [16] Foy et al. also studied the role of mICAM-1 in neutrophil adhesion to the endothelium with or without TNF- $\alpha$ stimulation in cremaster muscle.[19] They found that mICAM-1 is a ligand for neutrophil adhesion to the unstimulated endotheli$\mathrm{um}$, but that it is not a ligand for neutrophil adhesion to the TNF- $\alpha$-stimulated endothelium despite high ICAM-1 expression. It is unclear if these results are applicable to neutrophil recruitment in general, or if they only apply to some tissues including cremaster muscle, because the in vitro study using a flow chamber assay by Green et al. showed that LFA-1: ICAM-1 interaction is required for neutrophil adhesion to the IL-1 $\beta$ stimulated endothelium.[20] Of note, the endothelial cells of skeletal muscle beds have little in common with endothelial cells of the lung, liver or kidney, supporting the possibility that mICAM-1 may play a different role in neutrophil adhesion, depending on the tissues in which it is expressed. [19] A deficiency of mICAM-1 hinders recruitment, but increased expression of ICAM-1 during inflammation is not associated with enhanced neutrophil recruitment. The in vitro study by Yang et al. did not find any change in the degree of neutrophil transmigration in TNF- $\alpha$-activated endothelium.[21] Interestingly, however, their study demonstrated that the mode of transmigration changes with higher ICAM-1 expression. Generally, neutrophils can transmigrate between endothelial cells (paracellular migration) and through the endothelial cells (transcellular migration). Increased ICAM-1 expression facilitated more transcellular migration. Overall, ICAM-1 plays a significant role in neutrophil recruitment, but whether or not it plays a different role in different tissues needs further clarification.

\section{ICAM-1 Isoforms}

Several studies have shown that ICAM-1 isoforms are functional in vivo. [22] These studies were performed using ICAM-1 transgenic mice that were intended to be knockout mice by deleting one exon in their 
ICAM-1 gene. However, instead of serving as ICAM1 knockout mice, these exon-deletion transgenic mice expressed ICAM-1 isoforms. Exon 4 deletion mice express D1D4D5, D1D2D5 and D1D5, while exon 5 deletion mice express D1D2D3D5, D1D2D5 and D1D5. In the shock model using intraperitoneal injection of lipopolysaccharide (LPS), Robledo et al. found that wild type mice had a $50 \%$ survival rate, while exon 5-deletion mice showed a $23 \%$ survival rate, and $100 \%$ of exon 4-deletion mice survived. [23] Among exon 5-deletion mice, a large amount of neutrophil recruitment was observed in the liver, while neutrophil recruitment to the liver was minimal among exon 4-deletion mice. The distribution of these ICAM-1 isoforms among different tissues and their functions in relation to LFA-1 and Mac-1 needs further investigation, but this study suggests that ICAM-1 isoforms have an important role in neutrophil recruitment. Although full-length mICAM-1 expression is inducible, it is not known whether expression of ICAM-1 isoforms is inducible.

\section{Soluble ICAM-1 (sICAM-1)}

sICAM-1 can shed from the cell surface via a disintegrin and metalloproteinase (ADAM)-17 and neutrophil elastase (NE). sICAM-1 can exist as a monomer or as a dimer.[6] The binding strength of sICAM-1 to LFA-1 significantly depends on its dimerization. Dimer sICAM-1 showed 10-100 times as high binding affinity to LFA-1 as monomer sICAM-1.[14] The binding of sICAM-1 to Mac-1 has not yet been reported, but our experiments did not find significant binding between them. Various investigators reported that sICAM-1 competitively inhibited LFA1: ICAM-1 interaction. [24-26] Rieckmann et al. demonstrated that sICAM-1 at $150-200 \mathrm{ng} / \mathrm{mL}(\sim 3-4$ $\mathrm{nM}$ ) inhibited the binding of peripheral blood mononuclear cells (PBMCs) to cerebral endothelial cells [24], but it is unclear if the SICAM-1 was a dimer or a monomer. In addition, they showed that serum of patients suffering from multiple sclerosis inhibited the binding as well, suggesting that SICAM-1 in the human body could act as an inhibitor. The subsequent study by Meyer et al. examined the effect of sICAM-1 (recombinant sICAM $_{453}$ ) on the adhesion of SKW cells (LFA-1 expressing cells) to ICAM-1 and RAJI cell aggregation, and found that SICAM $_{453}$ inhibited LFA-1: ICAM-1 interaction with the $\mathrm{IC}_{50}$ of 20-40 $\mu \mathrm{M}$.[25] However, the authors recognized an accidental mutation in the recombinant SICAM-1, and also this sICAM-1 may have been expressed as a monomer. By contrast, the study by Jun et al. showed that dimer sICAM-1 had $\mathrm{IC}_{50}$ of 800 nM.[26] Overall, a wide range of $\mathrm{IC}_{50} \mathrm{~s}$ have been reported. This could be due to the abundance of dimerized SICAM- 1 in the prepared solution, as well as other experimental conditions. Because the concentration of circulating sICAM-1 in human subjects is around $500 \mathrm{ng} / \mathrm{mL}$ $(\sim 10 \mathrm{nM})$ [27] and serum from patients with multiple sclerosis inhibited LFA-1: ICAM-1 interaction, it is likely that SICAM-1 acts as an inhibitor in vivo. Knowing that sICAM-1 binds to LFA-1, it needs to be determined whether SICAM-1 can inhibit the interaction of LFA-1 with all ligands.

\section{Perioperative implications of ICAM-1}

\section{The role of ICAM-1 in perioperative disease models}

Ischemia-reperfusion is observed in various surgeries as a part of surgical procedures or after relief of vascular obstruction. And an extreme neutrophil recruitment as a result contributes to a tissue injury. The role of ICAM-1 in ischemia-reperfusion injury has been studied in various organs in animal models. A 45-minute of liver ischemia followed by reperfusion induced liver injury in rats, which was attenuated by ICAM-1 neutralizing antibody. (29) Similarly a 30-minute of myocardial ischemia by ligation of a coronary artery followed by reperfusion caused a significant myocardial necrosis in wild-type mice, while ICAM-1 knockout mice had less necrotic lesion.(30) The study by Kusterer et al. demonstrated that neutrophil adhesion following ischemia-reperfusion was attenuated by the administration of recombinant soluble ICAM-1 protein (31). Although not conclusive, Shimada et al. described the increased ICAM-1 expression in liver after partial hepatectomy than in liver prior to resection in patients, and suggested that this might be from ischemia-reperfusion injury as a result of Pringle maneuver for hepatectomy.(32) Of note, serum soluble ICAM-1 level was lower after resection than before resection, suggestion a possibility to attenuate liver injury with ICAM-1 as a target.

Future research directions of ICAM-1 in the perioper- 


\section{ative setting}

mICAM-1 undoubtedly plays a significant role by assisting leukocyte recruitment in the perioperative setting, but the role of mICAM-1 during various circumstances has yet to be investigated. Ischemia-reperfusion injury is such an example. The role of mI-

CAM-1 in leukocyte recruitment to the un-inflamed endothelium versus the inflamed endothelium in different tissues must be defined. Additionally, the role of mICAM-1 upregulation on the inflamed endothelium in various tissues has not been determined, although mICAM-1 is not a primary ligand in neutrophil adhesion to the inflamed endothelium in cremaster muscle. Because various pro-inflammatory cytokines are produced in the perioperative period, the endothelium in patients becomes inflamed. Some surgical procedures cause minimal stress responses, while procedures such as cardiac surgery in cardiopulmonary bypass can trigger an exaggerated pro-inflammatory response. This can cause a robust mICAM-1 upregulation on the endothelium. Although adequate neutrophil recruitment is beneficial for tissue health, an exaggerated accumulation of neutrophils is harmful. If mICAM-1 facilitates neutrophil recruitment to the significantly inflamed endothelium in certain tissues, this can lead to tissue destruction.

In order to study the role of mICAM-1 in these processes, the level of mICAM-1 expression must be determined. Because it is unrealistic to measure the levels of mICAM-1 levels on the endothelium from a clinical standpoint, an alternative method is warranted. sICAM-1 is easily measured by obtaining serum or plasma. Zonneveld et al. proposed a model of changes to mICAM-1 and sICAM-1 during progression of inflammatory responses [28]. Inflammation increases cell-surface expression of mICAM-1. Subsequently the level of mICAM-1 decreases during the resolution of inflammation as SICAM-1 increases. If aberrant shedding occurs along with sustained inflammation, the sICAM-1 level may not increase significantly. The level of sICAM-1 depends on the expression level of mICAM-1 and cleavage by sheddases such as ADAM17 and NE. This may explain why the level of sICAM-1 does not always correlate with disease outcomes in various disease processes, including sepsis.[28] In order to predict mICAM-1 expression levels, it is critical to understand expression levels of sICAM1, ADAM17, and NE.

Once these questions are answered, we need to understand if we can modulate mICAM-1 function. The administration of sICAM-1 may modulate mICAM-1 functions related to LFA-1. Less invasive surgical procedures and pharmacological interventions are likely candidates for modulating the function of mICAM-1. Although ICAM-1 has been studied for about 30 years since its discovery, there are still many questions to investigate. The elucidation of these questions, however, will allow us to understand patients' immune function more thoroughly and therefore opens a pathway to modulate those functions for patient benefit.

\section{Conflict Interests Disclosure:}

The authors have no conflicting interests to disclose.

Corresponding Author:Koichi Yuki, MD.

Department of Anesthesiology, Perioperative and Pain Medicine, Boston Children's Hospital, 300 Longwood Avenue, Boston, MA, 02115, USA. Tel: 1-617-3556225

\section{Email: koichi.yuki@childrens.harvard.edu}

Editor: Renyu Liu, MD; PhD. Associate Professor, Director of Preoperative Medicine, Department of Anesthesiology and Critical Care; Perelman School of Medicine at the University of Pennsylvania, 336 John Morgan building, 3620 Hamilton Walk, Philadelphia, PA 19104 . Phone: 2157461485 ; FAX: 2153495078

liur@uphs.upenn.edu

\section{Disclosure of Funding}

NIGMS GM101345 (K.Y.) and CHMC Anesthesia Foundation (K.Y.)

\section{Additional publication details}

Journal short name: Transl Perioper \& Pain Med

Received Date:Feb 29, 2016

Accepted Date: March 1, 2016

Published Date: March 26, 2016

Transl Perioper \& Pain Med 2016; 1(2):17-23

Citation and Copyright

Citation: Greenwald E, Yuki K. A translational consideration of Intercellular adhesion molecule-1 biology in the perioperative setting.Transl Perioper \& Pain Med 2016; 1(2):17-23 
Copyright: (C) 2016 Greenwald E. et al. This is an open-access article distributed under the terms of the Creative Commons Attribution License, which permits unrestricted use, distribution, and reproduction in any medium, provided the original author and source are credited.

\section{References}

1. Langer HF, Chavakis T. Leukocyte-endothelial interactions in inflammation. J Cell Mol Med. 2009;13(7):1211-20. Epub 2009/06/23. doi: JCMM811 [pii]

10.1111/j.1582-4934.2009.00811.x. PubMed PMID: 19538472; PubMed Central PMCID: PMC2861890.

2. Staunton DE, Marlin SD, Stratowa C, Dustin ML, Springer TA. Primary structure of ICAM-1 demonstrates interaction between members of the immunoglobulin and integrin supergene families. Cell. 1988;52(6):925-33. Epub 1988/03/25. doi: 0092-8674(88)90434-5 [pii]. PubMed PMID: 3349522.

3. Otto VI, Damoc E, Cueni LN, Schurpf T, Frei R, Ali S, et al. N-glycan structures and N-glycosylation sites of mouse soluble intercellular adhesion molecule-1 revealed by MALDI-TOF and FTICR mass spectrometry. Glycobiology. 2006;16(11):103344. Epub 2006/08/01. doi: cwl032 [pii] 10.1093/glycob/cwl032. PubMed PMID: 16877748.

4. King PD, Sandberg ET, Selvakumar A, Fang P, Beaudet AL, Dupont B. Novel isoforms of murine intercellular adhesion molecule-1 generated by alternative RNA splicing. J Immunol. 1995;154(11):6080-93. Epub 1995/06/01. PubMed PMID: 7751650 .

5. Dustin ML, Rothlein R, Bhan AK, Dinarello CA, Springer TA. Induction by IL 1 and interferon-gamma: tissue distribution, biochemistry, and function of a natural adherence molecule (ICAM-1). J Immunol. 1986;137(1):245-54. Epub 1986/07/01. PubMed PMID: 3086451.

6. Witkowska AM, Borawska MH. Soluble intercellular adhesion molecule-1 (sICAM-1): an overview. Eur Cytokine Netw. 2004;15(2):91-8. Epub 2004/08/21. PubMed PMID: 15319166.

7. Hogg N, Stewart MP, Scarth SL, Newton R, Shaw JM, Law SK, et al. A novel leukocyte adhesion deficiency caused by expressed but nonfunctional beta2 integrins Mac-1 and LFA-1. J Clin Invest. 1999;103(1):97-106. Epub 1999/01/12. doi: 10.1172/ JCI3312. PubMed PMID: 9884339; PubMed Central PMCID: PMC407855.

8. Fischer A, Lisowska-Grospierre B, Anderson DC, Springer TA. Leukocyte adhesion deficiency: molecular basis and functional consequences. Immunodefic Rev. 1988;1(1):39-54. Epub 1988/01/01. PubMed PMID: 3078709.
9. Hildebrand F, Pape HC, Harwood P, Muller K, Hoevel P, Putz C, et al. Role of adhesion molecule ICAM in the pathogenesis of polymicrobial sepsis. Exp Toxicol Pathol. 2005;56(45):281-90. Epub 2005/04/09. doi: S0940-2993(04)00068-5 [pii]10.1016/j.etp.2004.09.004. PubMed PMID: 15816357.

10. Nishida N, Xie C, Shimaoka M, Cheng Y, Walz $\mathrm{T}$, Springer TA. Activation of leukocyte beta 2 integrins by conversion from bent to extended conformations. Immunity. 2006;25(4):583-94. Epub 2006/10/19. doi: S10747613(06)00427-4 [pii] 10.1016/j.immuni.2006.07.016. PubMed PMID: 17045822.

11. Sen M, Yuki K, Springer TA. An internal ligand-bound, metastable state of a leukocyte integrin, alphaXbeta2. J Cell Biol. 2013;203(4):629-42. Epub 2014/01/05. doi: jcb.201308083 [pii] 10.1083/jcb.201308083. PubMed PMID: 24385486; PubMed Central PMCID: PMC3840939.

12. Carbo C, Yuki K, Demers M, Wagner DD, Shimaoka M. Isoflurane inhibits neutrophil recruitment in the cutaneous Arthus reaction model. J Anesth. 2013;27(2):261-8. Epub 2012/10/26. doi: 10.1007/s00540-012-1508-1. PubMed PMID: 23096126; PubMed Central PMCID: PMC3568683.

13. Li N, Mao D, Lu S, Tong C, Zhang Y, Long M. Distinct binding affinities of Mac-1 and LFA-1 in neutrophil activation. $\mathrm{J}$ Immunol. 2013;190(8):4371-81. Epub 2013/03/22. doi: jimmunol.1201374 [pii] 10.4049/jimmunol.1201374. PubMed PMID: 23514737.

14. Jun CD, Shimaoka M, Carman CV, Takagi J, Springer TA. Dimerization and the effectiveness of ICAM-1 in mediating LFA-1-dependent adhesion. Proc Natl Acad Sci U S A. 2001;98(12):6830-5. Epub 2001/06/08. doi: 10.1073/ pnas. 121186998 98/12/6830 [pii]. PubMed PMID: 11391003; PubMed Central PMCID: PMC34438.

15. Diamond MS, Staunton DE, Marlin SD, Springer TA. Binding of the integrin Mac-1 (CD11b/CD18) to the third immunoglobulin-like domain of ICAM-1 (CD54) and its regulation by glycosylation. Cell. 1991;65(6):961-71. Epub 1991/06/14. doi: 0092-8674(91)90548-D [pii]. PubMed PMID: 1675157.

16. Dunne JL, Collins RG, Beaudet AL, Ballantyne CM, Ley K. Mac-1, but not LFA-1, uses intercellular adhesion molecule-1 to mediate slow leukocyte rolling in TNF-alpha-induced inflammation. J Immunol. 2003;171(11):6105-11. Epub 2003/11/25. PubMed PMID: 14634125.

17. Dunne JL, Ballantyne CM, Beaudet AL, Ley K. Control of leukocyte rolling velocity in TNF-alpha-induced inflammation by LFA-1 and Mac-1. Blood. 2002;99(1):336-41. Epub 2002/01/05. PubMed PMID: 11756189.

18. Ding ZM, Babensee JE, Simon SI, Lu H, Perrard JL, Bullard DC, et al. Relative contribution of LFA-1 and Mac-1 to neutrophil adhesion and migration. J Immunol. 1999;163(9):5029-38. Epub 1999/10/21. doi: ji_v163n9p5029 [pii]. PubMed PMID: 10528208. 
19. Foy DS, Ley K. Intercellular adhesion molecule-1 is required for chemoattractant-induced leukocyte adhesion in resting, but not inflamed, venules in vivo. Microvasc Res. 2000;60(3):249-60. Epub 2000/11/18. doi: 10.1006/ mvre.2000.2272 S0026-2862(00)92272-6 [pii]. PubMed PMID: 11078641 .

20. Green CE, Schaff UY, Sarantos MR, Lum AF, Staunton DE, Simon SI. Dynamic shifts in LFA-1 affinity regulate neutrophil rolling, arrest, and transmigration on inflamed endothelium. Blood. 2006;107(5):2101-11. Epub 2005/11/05. doi: 200506-2303 [pii] 10.1182/blood-2005-06-2303. PubMed PMID: 16269618; PubMed Central PMCID: PMC1895714.

21. Yang L, Froio RM, Sciuto TE, Dvorak AM, Alon R, Luscinskas FW. ICAM-1 regulates neutrophil adhesion and transcellular migration of TNF-alpha-activated vascular endothelium under flow. Blood. 2005;106(2):584-92. Epub 2005/04/07. doi: 2004-12-4942 [pii] 10.1182/blood-2004-12-4942. PubMed PMID: 15811956; PubMed Central PMCID: PMC1635241.

22. Ramos TN, Bullard DC, Barnum SR. ICAM-1: isoforms and phenotypes. J Immunol. 2014;192(10):4469-74. Epub 2014/05/06. doi: 192/10/4469 [pii]

10.4049/jimmunol.1400135. PubMed PMID: 24795464; PubMed Central PMCID: PMC4015451.

23. Robledo O, Papaioannou A, Ochietti B, Beauchemin C, Legault D, Cantin A, et al. ICAM-1 isoforms: specific activity and sensitivity to cleavage by leukocyte elastase and cathepsin G. Eur J Immunol. 2003;33(5):1351-60. Epub 2003/05/06. doi: 10.1002/eji.200323195. PubMed PMID: 12731061.

24. Rieckmann P, Michel U, Albrecht M, Bruck W, Wockel L, Felgenhauer K. Soluble forms of intercellular adhesion molecule-1 (ICAM-1) block lymphocyte attachment to cerebral endothelial cells. J Neuroimmunol. 1995;60(1-2):9-15. Epub 1995/07/01. PubMed PMID: 7642752.

25. Meyer DM, Dustin ML, Carron CP. Characterization of intercellular adhesion molecule-1 ectodomain (sICAM-1) as an inhibitor of lymphocyte function-associated molecule-1 interaction with ICAM-1. J Immunol. 1995;155(7):3578-84. Epub 1995/10/01. PubMed PMID: 7561056.

26. Jun CD, Carman CV, Redick SD, Shimaoka M, Erickson HP, Springer TA. Ultrastructure and function of dimeric, soluble intercellular adhesion molecule-1 (ICAM-1). J Biol Chem. 2001;276(31):29019-27. Epub 2001/06/08. doi: 10.1074/jbc. M103394200 M103394200 [pii]. PubMed PMID: 11390397.

27. Woska JR, Jr., Morelock MM, Jeanfavre DD, Bormann BJ. Characterization of molecular interactions between intercellular adhesion molecule-1 and leukocyte function- associated antigen-1. J Immunol. 1996;156(12):4680-5. Epub 1996/06/15. PubMed PMID: 8648112.

28. Zonneveld R, Martinelli R, Shapiro NI, Kuijpers TW, Plotz FB, Carman CV. Soluble adhesion molecules as markers for sepsis and the potential pathophysiological discrepancy in neonates, children and adults. Crit Care. 2014;18(1):204. Epub 2014/03/08. doi: cc13733 [pii] 10.1186/cc13733. PubMed PMID: 24602331 ; PubMed Central PMCID: PMC4014977. 